\title{
POSITIVE DERIVATIONS ON PARTIALLY ORDERED LINEAR ALGEBRA WITH AN ORDER UNIT
}

\author{
TAEN-YU DAI AND RALPH DEMARR
}

\begin{abstract}
We show that the range of a positive derivation on a Dedekind $\sigma$-complete partially ordered linear algebra with an order unit is a set of generalized nilpotents. With additional assumptions on the algebra, we show that the algebra has an important property similar to a property of the algebra of upper triangular matrices.
\end{abstract}

1. Introduction and definitions. Derivations on operator algebras have been studied extensively by various authors; see [4]. DeMarr has shown that every Banach algebra of bounded linear operators can be ordered so that it becomes a partially ordered linear algebra with an order unit in which norm convergence and order convergence are equivalent [3, Theorem, p. 637]. This motivates the study of positive derivations on Dedekind $\sigma$-complete partially ordered linear algebra (dsc-pola) with an order unit.

A dsc-pola, denoted by $A$, is a real linear associative algebra which satisfies the following two conditions: (1) It is partially ordered so that it is a directed partially ordered linear space and $0 \leqslant x y$ whenever $0 \leqslant x, y \in A$. (2) It is Dedekind $\sigma$-complete, i.e., if $x_{n} \in A$ and $x_{1} \geqslant x_{2} \geqslant \cdots \geqslant 0$, then $\inf \left\{x_{n}\right\}$ exists. A dsc-pola has the Archimedean property: if $x, y \in A$ and $\alpha x \leqslant y$ for all positive real numbers $\alpha$, then $x \leqslant 0$. In this paper, we assume $A$ has a multiplicative identity $1 \geqslant 0$. Let $I=\left\{y \in A: y \geqslant 1\right.$ and $\left.y^{-1} \geqslant 0\right\}$ and then define $A_{1}=I-I$. It is shown in [1] that $A_{1}$ is an order-closed and orderconvex commutative subalgebra of $A$ which behaves much like an algebra of real-valued functions. We call $A_{1}$ the diagonal or functional part of $A$. For a detailed discussion and examples of $A_{1}$, see [1]; there we used the term polac instead of dsc-pola.

We say that $u \in A$ is an order unit if for each $x \in A$ there exists a real number $\delta$ such that $-\delta u \leqslant x \leqslant \delta u$. The fact that $A$ has an order unit is important in this paper. Example 3.5 shows that our main Theorem 2.3 is not true without an order unit.

A derivation $f$ on $A$ is a linear map from $A$ into itself such that $f(x y)=$ $x f(y)+f(x) y$ for all $x, y$ in $A$. A positive derivation has the additional property that $f(x) \geqslant 0$ whenever $x \geqslant 0$. An inner derivation is a derivation

Received by the editors October 31, 1977 and, in revised form, March 20, 1978.

AMS (MOS) subject classifications (1970). Primary 06A70; Secondary 47B55, 15 A45.

Key words and phrases. Dedekind $\sigma$-complete partially ordered linear algebra, order unit, derivation, generalized nilpotent, matrix algebra, diagonal projection map. 
having the form $f(x)=x a-a x$ for all $x \in A$, where $a$ is some fixed element in $A$.

Examples of order units and positive derivations are given in $\S 3$.

In [2], a diagonal projection map is defined to be a positive linear map $\Delta$ from $A$ into $A_{1}$ such that $\Delta(1)=1$ and $0 \leqslant \Delta(x) \leqslant x$ whenever $0 \leqslant x$. This map was used to study the structure of $A$. For a detailed discussion and examples of $\Delta$, see [2].

A generalized nilpotent is an element $z$ in $A$ such that for each $\alpha>0$ there exists $v \in A$, depending on $\alpha$, satisfying the inequality $-v \leqslant \alpha^{n} z^{n} \leqslant v$ for all positive integers $n$. Note that every nilpotent in $A$ is a generalized nilpotent.

Lemma 1.1. If $y, z \in A$ and $-z \leqslant y \leqslant z$, then $-z^{n} \leqslant y^{n} \leqslant z^{n}$ for all positive integers $n$. Hence, if $z$ is a generalized nilpotent, then $y$ is also a generalized nilpotent. In particular, if $z$ is a nilpotent, then $y$ too is a nilpotent.

The proof is left as an exercise for the reader.

2. Main results. We assume that $A$ has an order unit and that $f$ is a positive linear map from $A$ into itself which satisfies the inequality $f(x y) \geqslant x f(y)+$ $f(x) y$ whenever $x \geqslant 0$ and $y \geqslant 0$. This not only describes a positive derivation, but it also enables us to study a certain kind of homomorphism which is discussed in Example 3.3. Although an order unit is not unique, in this paper it is desirable to choose an order unit $u$ such that $u \leqslant u^{2} \leqslant \beta u$, where $\beta \geqslant 1$ and $\beta$ is close to 1 . The range of $f$ is described in the following theorems.

THeOREM 2.1. If $u \in A$ is an order unit such that $u \leqslant u^{2} \leqslant \beta u$, where $1 \leqslant \beta<2$, then $f(x)=0$ for all $x \in A$.

Proof. Since $u$ is an order unit and $f$ is a positive linear map, we need only show that $f(u)=0$. Let $z=f(u)$. First applying $f$ to both sides of the inequality $u^{2} \leqslant \beta u$, then using the definition of $f$, we obtain $u z+z u \leqslant f\left(u^{2}\right)$ $\leqslant \beta z$. Thus, $2 u z u \leqslant u^{2} z u+u z u^{2} \leqslant \beta u z u$. This implies that $(\beta-2) u z u \geqslant 0$. Since $\beta<2$, it follows that $0 \geqslant u z u \geqslant 0$ or $u z u=0$. Since $u$ is an order unit, we can find some number $\delta$ such that $\delta u \geqslant 1$. Now clearly we have $0=$ $(\delta u) z(\delta u) \geqslant z \geqslant 0$. Therefore, $z=0$.

THEOREM 2.2. If $u \in A$ is an order unit such that $u \leqslant u^{2} \leqslant 2 u$, then $f(x)^{2}=0$ for all $x \in A$.

Proof. Let $z=f(u)$. We first show that $z^{2}=0$. As in Theorem 2.1, we obtain $2 u z u \leqslant u^{2} z u+u z u^{2} \leqslant 2 u z u$. Thus, $2 u z u=u^{2} z u+u z u^{2}$ or $0 \leqslant\left(u^{2}-\right.$ $u) z u=u z\left(u-u^{2}\right) \leqslant 0$. Hence, $\left(u^{2}-u\right) z u=0$. Since $\left(u^{2}-u\right) z>0$ and $\delta u \geqslant 1$ for some $\delta>0$, we get $0=\left(u^{2}-u\right) z(\delta u) \geqslant\left(u^{2}-u\right) z \geqslant 0$ or $\left(u^{2}-\right.$ $u) z=0$. It follows that $0=f\left(\left(u^{2}-u\right) z\right) \geqslant\left(u^{2}-u\right) f(z)+f\left(u^{2}-u\right) z \geqslant 0$. Since both terms $f\left(u^{2}-u\right) z$ and $\left(u^{2}-u\right) f(z)$ are nonnegative, we have 
$0=f\left(u^{2}-u\right) z=\left(f\left(u^{2}\right)-z\right) z=f\left(u^{2}\right) z-z^{2} \geqslant(u z+z u) z-z^{2}$. Hence, $u z^{2}+z u z \leqslant z^{2}$. Multiplying by $u$ on both sides of the last inequality, we obtain $u^{2} z^{2}+(u z)^{2} \leqslant u z^{2} \leqslant u^{2} z^{2}$. Consequently, $(u z)^{2}=0$. From $\delta u \geqslant 1$ we know that $\delta u z \geqslant z \geqslant 0$, so that $z^{2}=0$. Now for any $x \in A$ we have $-\alpha u \leqslant$ $x \leqslant \alpha u$ for some $\alpha$. Thus, $-\alpha z \leqslant f(x) \leqslant \alpha z$. Using Lemma 1.1 and the fact that $z^{2}=0$, we conclude that $f(x)^{2}=0$.

THEOREM 2.3. If $u \in A$ is an order unit such that $u \leqslant u^{2} \leqslant \beta u$, where $\beta>2$, then $f(x)$ is a generalized nilpotent for all $x \in A$.

Proof. Again, we need only show that $z=f(u)$ is a positive generalized nilpotent. From $u z+z u \leqslant f\left(u^{2}\right) \leqslant \beta z$ we see that $f(u) z+z f(u) \leqslant f(u z+$ $z u) \leqslant \beta f(z)$ or $2 z^{2} \leqslant \beta f(z)$. By induction we will show that $2 n z^{n+1} \leqslant$ $\beta f\left(z^{n}\right)$ for every positive integer $n$. We have proved the inequality is true for $n=1$. Assume the inequality is true when $n=k$. Hence, $\beta f\left(z^{k+1}\right) \geqslant \beta f\left(z^{k}\right) z$ $+\beta z^{k} f(z) \geqslant 2 k z^{k+1} z+2 z^{k} z^{2}=2(k+1) z^{k+2}$. Thus, the induction is complete.

Let $1 \leqslant \lambda$ be a number such that $z \leqslant \lambda u$. Next, using induction again we will show that $n ! z^{n} \leqslant(\beta \lambda)^{n} u$ for every positive integer $n$. Clearly the inequality is true for $n=1$. Suppose the inequality holds for $n=k$. Hence, $k ! \beta f\left(z^{k}\right) \leqslant \beta(\beta \lambda)^{k} f(u) \leqslant \beta(\beta \lambda)^{k} \lambda u=(\beta \lambda)^{k+1} u$. From the previous paragraph we have $\beta f\left(z^{k}\right) \geqslant 2 k z^{k+1} \geqslant(k+1) z^{k+1}$; therefore, $(\beta \lambda)^{k+1} u \geqslant$ $k !(k+1) z^{k+1}=(k+1) ! z^{k+1}$. Thus, the second induction is complete. Now for any number $\alpha>0$ we see that $n ! \alpha^{n} z^{n} \leqslant(\alpha \beta \lambda)^{n} u$ or $0 \leqslant \alpha^{n} z^{n} \leqslant$ $(\alpha \beta \lambda)^{n} u / n$ ! for all positive integers $n$. Since $\left\{(\alpha \beta \lambda)^{n} / n !\right\}$ is a bounded sequence, there exists a real number $\gamma$ such that $0 \leqslant \alpha^{n} z^{n} \leqslant \gamma u$ for all $n$ (of course, $\gamma$ depends on $\alpha$ ). This shows that $z$ is a positive generalized nilpotent. Since $u$ is an order unit and $f$ is a positive linear map, for any $x \in A$ there exists a number $\delta$ such that $-\delta u \leqslant x \leqslant \delta u$ and therefore $-\delta z \leqslant f(x) \leqslant \delta z$. Using Lemma 1.1 and the fact that $z$ is a positive generalized nilpotent, we may conclude that $f(x)$ is a generalized nilpotent.

Conditions were given in [2] which imply that a diagonal projection map $\Delta$ is multiplicative; that is, $\Delta(x y)=\Delta(x) \Delta(y)$ for all $x, y$ in $A$. Roughly speaking, if $A$ has a diagonal projection map $\Delta$ which is multiplicative, then $A$ is like an algebra of upper triangular matrices. The examples in the following section will illustrate this. The reader should observe the following interesting points. In Example 3.1, the matrix $1+z(z=f(u))$ is not an order unit of $A$; in fact $1+z=1$. Note that $\Delta$ is not multiplicative. However, in Examples $3.2,3.3$, and 3.4, the matrix $1+z$ is an order unit. Note that $\Delta$ is multiplicative in each case. These observations lead to the following theorem.

TheOREM 2.4. If a diagonal projection map $\Delta$ exists on $A$ and if $1+z$ $(z=f(u), u$ as in Theorem 2.3) is an order unit, then $\Delta$ is multiplicative. 
Proof. We first show that $\Delta(z)=\Delta\left(z^{2}\right)=0$. Since $z$ is a positive generalized nilpotent, for each $\alpha>0$ there exists $v \in A$ such that $0 \leqslant \alpha^{n} z^{n} \leqslant v$ for all positive integers $n$. Using Lemma 3.4 of [2], we have $0 \leqslant \Delta\left(\alpha^{n} z^{n}\right)=$ $\alpha^{n} \Delta\left(z^{n}\right) \leqslant 1$ for all $n$. Since this inequality holds for arbitrary $\alpha>0$ and since $A$ has the Archimedean property, we conclude that $\Delta\left(z^{n}\right)=0$ for all $n$; in particular, $\Delta(z)=\Delta\left(z^{2}\right)=0$.

By Lemma 4.1 of [2], to prove that $\Delta$ is multiplicative we need only verify that if $0 \leqslant w \in A$ and $\Delta(w)=0$, then $\Delta\left(w^{2}\right)=0$.

Since $1+z$ is an order unit, we can find some $\delta>0$ such that $0 \leqslant w \leqslant$ $\delta(1+z)$. By the fact that $\Delta(z)=0$ and by the definition of $\Delta$, we see that $0 \leqslant \delta(1+z)-w-\Delta(\delta(1+z)-w)=\delta z-w$. Hence, $0 \leqslant w^{2} \leqslant \delta^{2} z^{2}$. Let us apply $\Delta$ throughout the last inequality. The fact that $\Delta$ is a positive linear map gives $0 \leqslant \Delta\left(w^{2}\right) \leqslant \delta^{2} \Delta\left(z^{2}\right)=0$. Therefore, $\Delta\left(w^{2}\right)=0$ which means that $\Delta$ is multiplicative.

\section{Examples.}

EXAMPLE 3.1. Let $A$ be the matrix algebra of all $m$-by- $m$ real matrices with componentwise ordering. Hence, $A$ is a dsc-pola. If $u$ is the matrix with all entries equal to $1 / m$, then $u$ is an order unit. Since $u=u^{2}$, we have $\beta=1$. By Theorem 2.1 we conclude that the only positive derivation on $A$ is the zero derivation. It is easy to check that in this example $A_{1}$ is the set of all diagonal matrices in $A$. For $x \in A$ define $\Delta(x)$ to be the diagonal part of $x$. We can easily check that $\Delta$ is a diagonal projection map which is not multiplicative.

EXAMPLE 3.2. Let $A$ be the matrix algebra of all $m$-by- $m$ real upper triangular matrices with componentwise ordering. Hence, $A$ is a dsc-pola. The matrix $u$ in $A$ with diagonal entries equal to 1 and $\varepsilon>0$ elsewhere is an order unit. Note that $u \leqslant u^{2} \leqslant \beta u$, where $\beta=2+(m-2) \varepsilon$. If $m=2$, then $\beta=2$. Take the diagonal matrix $a=\left[i \delta_{i j}\right]$, where $\delta_{i j}$ is the Kronecker delta and define $f(x)=x a-a x$ for $x \in A$. It is easy to check that $f$ is a positive inner derivation and that $f(x)^{m}=0$ for all $x \in A$. The set $A_{1}$ is the collection of all diagonal matrices in $A$. If we define $\Delta$ as in Example 3.1 , then $\Delta$ is a multiplicative diagonal projection map.

Example 3.3. Let $A$ be the dsc-pola described in Example 3.2. Define $g(x)=a^{-1} x a$, where $x \in A$ and $a$ is the same diagonal matrix as in Example 3.2. We can verify easily that $g$ is linear, $g(x y)=g(x) g(y)$ for all $x, y \in A$ and $g(x) \geqslant x \geqslant 0$ whenever $x \geqslant 0$. Let $f$ be a linear map on $A$ defined by $f(x)=g(x)-x$. Note that for $0 \leqslant x, y$ we have $f(x) \geqslant 0, f(y) \geqslant 0$ and

$$
\begin{aligned}
f(x y) & =g(x y)-x y=g(x) g(y)-x y \\
& =(f(x)+x)(f(y)+y)-x y \geqslant x f(y)+f(x) y .
\end{aligned}
$$

This is an example in which the inequality can be strict. Furthermore, $f(x)^{m}=0$ for all $x \in A$.

In general, if $g(x)$ is a homomorphism of a dsc-pola $A$ into itself such that $g(x) \geqslant x$ for $x \geqslant 0$, then $f(x)=g(x)-x$ satisfies the conditions at the 
beginning of $\$ 2$. Theorem 2.3 shows that if $A$ has an order unit, then the difference $g(x)-x$ is a generalized nilpotent for all $x \in A$.

EXAMPLE 3.4. Let $A$ be the algebra of matrices of the form $x=\left[\begin{array}{ll}\alpha & \gamma \\ 0 & \alpha\end{array}\right]$, where $\alpha$ and $\gamma$ are reals. If $A$ is partially ordered componentwise, then $A$ is a dsc-pola with $u=\left[\begin{array}{ll}1 & 1 \\ 0 & 1\end{array}\right]$ as an order unit and $u<u^{2} \leqslant 2 u$. Since $A$ is commutative, there is no inner derivation on $A$, but the map $f(x)=\left[\begin{array}{ll}0 & \gamma \\ 0 & 0\end{array}\right]$ is a positive derivation. Note that $f(x)^{2}=0$. The set $A_{1}$ is the collection of all scalar matrices in $A$. If we define $\Delta$ as in Example 3.1 , then $\Delta$ is a multiplicative diagonal projection map.

EXAMPLE 3.5. Let $A$ be the algebra of all real polynomials of the form $x(\tau)=\alpha_{0}+\alpha_{1} \tau+\alpha_{2} \tau^{2}+\cdots+\alpha_{n} \tau^{n}$. The ordering $0 \leqslant x$ means that the coefficients of $x(\tau)$ are nonnegative. $A$ is a dsc-pola with no order unit. The ordinary derivative $f$ is a positive derivation. Let $y(\tau)=\tau$. We see that $f(y)=1$ is not a generalized nilpotent. This example shows that Theorem 2.3 is not true if $A$ is an arbitrary dsc-pola.

EXAMPLE 3.6. We now give an example in which $z=f(u)$ is a generalized nilpotent and not just a nilpotent. Let $B$ be the algebra of all infinite matrices $x=\left[\alpha_{i j}\right]$, where $i, j=1,2,3, \ldots$, which are in upper triangular form. This means that $\alpha_{i j}=0$ for $i>j$. If we partially order $B$ componentwise, then $B$ is a dsc-pola. Of course, $B$ does not have an order unit, but we may construct subalgebras of $B$ which do have order units. We do this by first selecting any matrix $s$ in $B$ such that $0 \leqslant s^{2} \leqslant s$ and then we define $u=1+s$. It is easy to show that $u \leqslant u^{2} \leqslant 3 u$. We next define

$$
A=\bigcup_{k=1}^{\infty}\{x:-k u \leqslant x \leqslant k u\} .
$$

Of course, $A$ is an order-convex subalgebra of $B$ which has $u$ as an order unit. Since $A$ is order-convex, it is also a dsc-pola. The reader should note that $A$ depends on $u$.

Let us now define the fixed diagonal matrix $a=\left[\alpha_{i} \delta_{i j}\right] \in B$, where $0 \leqslant \alpha_{1}<\alpha_{2}<\cdots \leqslant 1$, and then define the positive inner derivation $f(x)$ $=x a-a x$ for all $x \in B$. Since $0 \leqslant a \leqslant 1$, it follows that $f(x) \leqslant x$ whenever $0 \leqslant x$. Hence, we may regard $f(x)$ as a derivation which maps $A$ into itself.

In particular, let us select $s=\left[\sigma_{i j}\right] \in B$, where $\sigma_{i j}=2^{-j}$ whenever $1 \leqslant i \leqslant$ $j$. It is easy to verify that $0 \leqslant s^{2} \leqslant s$. Note that $f(s)$ has a positive offdiagonal entry in any position where $s$ has a positive off-diagonal entry. Using this fact and the fact that $z=f(u)=f(s)$, we see that $z$ is a generalized nilpotent, but $z^{n}$ is not zero for any positive integer $n$.

\section{REFERENCES}

1. T. Y. Dai, On some special classes of partially ordered linear algebras, J. Math. Anal. Appl. 40 (1972), 649-682.

2. T. Y. Dai and R. DeMarr, Partially ordered linear algebras with multiplicative diagonal 
projection map, Trans. Amer. Math. Soc. 224 (1976), $179-187$.

3. R. DeMarr, On partially ordering operator algebras, Canad. J. Math. 19 (1967), 636-643.

4. R. V. Kadison and J. R. Ringrose, Derivations and automorphisms of operator algebras, Comm. Math. Phys. 4 (1967), 32-63.

Department of Mathematics, York College (CUNY), Jamaica, New York 11451

Department of Mathematics, Universtty of New Mexico, AlbuQuerque, New Mexico 87131 